\title{
Integrating Antenatal and Postnatal Pregnancy Services to Hospital Management System
}

\author{
Yusuf Mohammed Danlami ${ }^{1}$, Abba Almu ${ }^{1}$, Hassan Mairoh ${ }^{1}$, Mohammed Kabiru Hamza ${ }^{1}$ \\ ${ }^{1}$ Usmanu Danfodiyo University \\ P. M. B. 2346, Sokoto, Nigeria
}

DOI: $10.22178 /$ pos.39-3

LCC Subject Category:

TK7885-7895

Received 01.08.2018

Accepted 15.09.2018

Published online 31.10.2018

Corresponding Author:

Mohammed Yusuf Danlami

mohammed.dyusuf@udusok.edu.ng

(C) 2018 The Authors. This article

is licensed under a Creative

Commons Attribution 4.0 License

(c) (1)

\begin{abstract}
Antenatal and postnatal processes and services is a component of Hospital Management Systems that has been given very little attention. Most healthcare institution carries out the antenatal and postnatal processes manually and keeps records of the whole processes on paper. In most cases in most hospital, records kept on paper and files get missing or misplaced leading to the loss of important records of clients which would have been very helpful for future pregnancies and clinical diagnosis. Report generation and statistical figures are difficult to generate with the manual based system. Data errors, security, and privacy are another problems associated with the manual system. In this paper, the antenatal and postnatal services were incorporated into the Hospital Management System of Usmanu Danfodiyo University, Sokoto with a view to reducing the issues and inconsistencies encountered with the manual procedure. Dreamweaver, PHP, CSS, JavaScript, Ajax, HTML and MySQL are the technological tools used to automate the incorporated antenatal and postnatal services in the system. The usefulness of the proposed system was evaluated using the System Usability Scale (SUS) questionnaire and some clinical users. The experimental evaluation shows that the developed system is beneficial to the clinic and the general public based on the result obtained in this study. The results also demonstrated that the developed system can fit into the antenatal and postnatal routine of many hospitals with little or no modification.
\end{abstract}

Keywords: pregnancy; antenatal services; postnatal services; hospital management; evaluation.

\section{INTRODUCTION}

Hospital systems are complex systems built in order to capture, store, organize, process and transmit patient's records and also to ease the various processes and activities within the hospital for the purpose of improving the quality, efficiency and performance of service delivery to the teaming population of patients within and outside the hospital [1]. Hospital Information systems automate the whole hospital processes so as to achieve the above mentioned requirements. These systems have various smaller subcomponents (systems) which when integrated together give rise to single large Hospital information systems. These smaller sub-components or systems include: Laboratory information system, Nursing Information System, Patient Record Information System, Pharmacy Information System and lots more [1]. These sub-systems if properly implemented will definitely lead to im- prove performance, quality and efficiency of the whole hospital operations and service delivery and satisfaction to patients.

The main purpose of any healthcare institution is to save lives by treating patients from various illnesses. These sub-components mentioned above of any hospital system work hand in hand with each other to ensure patients safety and health condition. A normal or basic hospital routine on the treatment of patients is for patients to see a doctor after booking an appointment, patient diagnosis by a doctor, doctor gives prescription to patient, doctor sends a patient to either pharmacy to get prescribed drugs or to the lab for tests, doctor can also admit patient and lots more. All these processes when carefully observed form the various sub-components of a hospital management system mentioned earlier. A clinical routine or component of a hospital management system often neglected and not 
been automated or integrated into most existing systems is the antenatal and postnatal care services for pregnant women. Antenatal care services are rendered to a pregnant woman throughout the pregnancy period until the day she gives birth [6]. This service is to ensure that the mother and the baby are in good condition, so as to ensure safe delivery of both mother and fetus. Postnatal service is rendered to a woman who has delivered successfully [3]. This service is to ensure the healthy growth of the baby from the time he/she is delivered to when he/she is about a year old. This is also to protect the baby from diseases that may hinder the healthy growth of the baby.

In this work, the antenatal and postnatal care services are automated and integrated within the normal hospital patient to doctor processes in order to provide a lot of benefits to the patients. The automation will help in the generation of reports to monitor the quality of care, efficiency, and effectiveness of pregnancy care. The reports that could be generated are: maternal or infant mortality, mortality rates, number of successful deliveries, women expected to deliver at a given month, a monthly rate of the antenatal and postnatal visit which will determine value it has to the community and a lot more of other reports not listed. Lecture given to pregnant women during the antenatal visit is automated as tutorials on web pages to educate women about pregnancy safety tips. This will benefit not only the registered pregnant women but also the community at large as anyone with access to the portal will benefit since it is online.

\section{RELATED WORKS}

Authors [4] developed a Mobile Phone Based Antenatal Care Support System (MPACSS) for pregnant women by considering the fact that mobile phones usage and access has globally exploded. The system is built based on an artificial intelligent tool-expert system that provides accurate decision-making process. The system Graphical User Interface (GUI) allows the patient to access the application using a mobile phone to get the lists of symptoms which the patients clicks or selects at a time. However, the system doesn't automate antenatally and postnatal routines, clinical test, report generation, and export. Also, the system is a native mobile application which is expensive to implement by other healthcare clin- ics and might not work on some categories of mobile phones.

Researches [2] proposed a decision support system IASHA to empower the Accredited Social Health Activist (ASHA) and Auxiliary Nurse Midwife (ANM) workers to provide efficient and transparent maternal healthcare services to pregnant women. ASHA are female health activist in every village who are selected by the village and accountable to it. ANM are female health workers in rural health sub-center responsible for registration and care for pregnant women in the community. Both ASHA and ANM workers will be provided with low cost android phones with IASHA application installed on it. IASHA is an intelligent healthcare system built to automate the workflow of ASHA and ANM workers. The IASHA application can be used to register pregnant women details, medical details, determine Expected Date of Delivery (EDD) of pregnant women, record vaccination records, lab tests etc. Immunization details (i.e. postnatal care) of a newborn child can also be recorded on IASHA. The IASHA application can also be used for accessing various patient conditions, questions and suggestions to diseases selected, generate reports and also determine if a pregnant woman can have a normal delivery based on symptoms on available records. This indicates that the IASHA application is been used for monitoring antenatal visits of a pregnant woman and also postnatal care for the newborn baby. However IASHA is only accessible to ASHA and ANM workers, training module 6 and 7are only available to ASAH and ANM workers, patients can't use the IASHA application for access to lectures on pregnant care, review their records and health status, reports not provided in a suitable format for export, printing of report is not expected on mobile phone except if a network printer is available. Also mobile applications are very expensive and difficult to maintain and adopt by other healthcare institution and works best if an existing application is available where the mobile app is an upgrade or supplementary addition to the existing application to support the healthcare process.

Author [5] proposed a mobile health system (mhealth) for pregnant women so as to overcome the weakness of the current Bangladeshi pregnancy monitoring system. The proposed system is a mobile-based as well as a web-based system. The aim of the proposed system is to provide access to healthcare services online, reduce mater- 
nal and infant mortality, improve on the existing system by incorporating email and SMS messages, enable local registration of pregnant women and improve effectiveness and ease of use. A pregnant woman registers on the system for antenatal and postnatal services by herself or by the health workers for these women who are not capable of registering themselves. Registration for a pregnant woman is valid until the age of the newborn is one year (i.e. after postnatal care services). Physical examination of pregnant women by doctors and nurses during registration and antenatal care is captured by the system. The m-health system has several modules accessible by doctors, nurses, patients, administrators, and others. Examples of some of the modules are: patient registration modules, doctor's module (where a doctor sees appointment from patients, gives suggestions to patients, book appointment with patients in case of complications etc), Laboratory module (where lab test can be performed on pregnant women and test results saved in a database) etc. The system is designed to archive pregnant women information when their registration becomes invalid or expires. However, the system is not completely a native mobile health system but a web system. Web application systems are accessible to mobile devices through the use of a web browser. Building a native mobile application for an antenatal and postnatal system is very expensive. Reports module is not considered fully by the system which indicates that export of such available reports in a suitable format is not taken into consideration for patients that can be referred to other healthcare due to complications, migration or travel.

Authors [7] proposed an Online Antenatal Consultation System (OACS) using Unified Modeling Language (UML) web engineering approach so as to reduce the rate of infant and maternal mortality in rural areas of Nigeria. The system is aimed at providing quality antenatal support to pregnant women in rural areas of Nigeria. It is accessible to pregnant women, doctors, nurses through the use of the following devices: PC, Desktop, Laptop, Palmtop, and mobile devices such as smartphones, tablets, and Android gadgets. The OACS Administrators manages the use and operation of the system by generating pins, reports of pins, users etc. these pins are required during by users during registration and login to the system. A patient can register herself or by the help of the staff nurses for those patients who can't register by themselves. A patient can have full access to her profile where she can book for an appointment with a doctor nurse, lay complaints, receives results and prescriptions online from a doctor or nurse. Doctors and nurses also must be registered on the system using pins generated and issued by the administrator. A doctor can view patients complains, offer prescription, give advice to patients, sends messages etc. Nurses can view appointments from patients and assigns an appointment with a doctor. The OCAS has a lot of weakness as it is only designed to provide support to pregnant women in rural areas who cannot have access to a primary healthcare facility due to the long distance. Women who do not have mobile phones or other devices to access the system cannot benefit from the system. Antenatal routine checkup, clinical test and physical evaluation by a doctor are not captured by the system. Therefore few reports can be generated whereby those relevant reports that can enhance decision making might not be captured or generated as reports. Reports generated cannot be exported into a suitable format for use. However, the security of the system is considered as a user must first register using secure pins generated by the Administrator and must log in after registration using a password before having access to the system.

\section{MATERIALS AND METHODS}

The Hospital Antenatal and Post Natal system is developed using build methodology. This will require the use of software development lifecycle tools such as requirement analysis, system design, implementation, testing, and deployment. The requirement analysis is conducted using the interview method of data collection. In the cause of the interview, a voice recorder was used to also capture voice discussions to ensure that all necessary details are not left out.

The system has been an online web application is implemented using tools and technologies such as Dreamweaver, WAMP Server, PHP, CSS, JavaScript, Ajax, HTML and MYSQL Database. The Dreamweaver is a text editor that enables the writing of codes for the system. It is a good tool used for writing codes with a lot of functionalities that make web programming easy. The WAMP Server is a bundle of servers for windows operating system. Other versions of WAMP Servers for other operating systems are the LAMP (Linux OS), MAMP (MAC OS) and XAMP (windows and Linux OS). MYSQL Database is used to store re- 
cords of client's antenatal visits, postnatal and vaccination of infants, login credentials and any other relevant data. The use of MYSQL Database makes the system to be built a dynamic web application. The PHP script is used for writing scripts to interact with our MYSQL Database. These interactions could be establishing connections to the database, retrieving or inserting records to the database and modifying or deleting records from the database. PHP is used to perform other functions and operations such as mathematical computation, export of records to files, getting date and time etc. HTML is used to structure content on the web page. That is placing of contents at various positions on the web page for display. HTML is used in designing forms which will capture data into our database or be used for login verification on the system. CSS is used to make the system more attractive and beautiful by adding colors, modifying contents (e.g. font style, font family, background color etc.) and make HTML elements move from one position to the other etc. Ajax and JavaScript are used to make the system more interactive and user-friendly. They can also be used for HTML form validations.

\section{SYSTEM ARCHITECTURE}

The system comprises of the following components to function effectively. These components are user authentication, access control, HMS ANC modules, ANC tutorial/e-resources modules, and a database component. The users of the system are the clients, clinic staff, nurses, doctors, the community and an administrator. All users of the system can access the lectures and e-resources modules without authentication as its free and open for everybody. Access to other modules requires authentication of the user and the right access to each module is controlled by the access control component of the system. No user can access the database component of the system directly except the system administrator (Figures 1, 2).

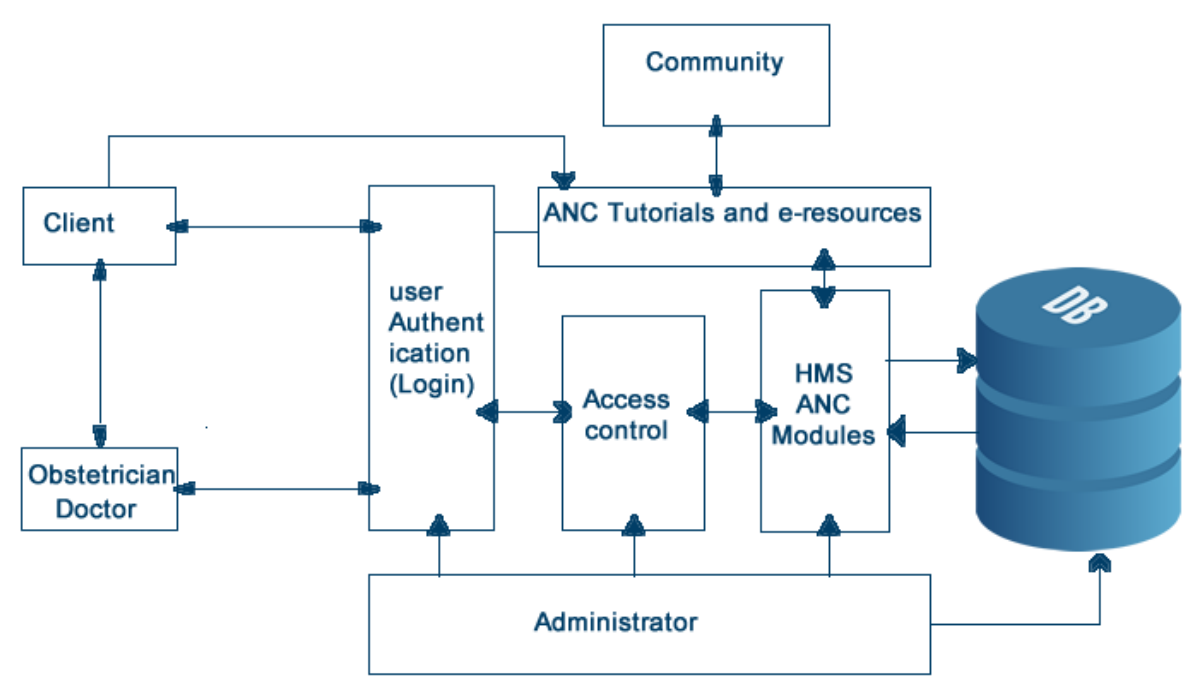

Figure 1 - System Architecture

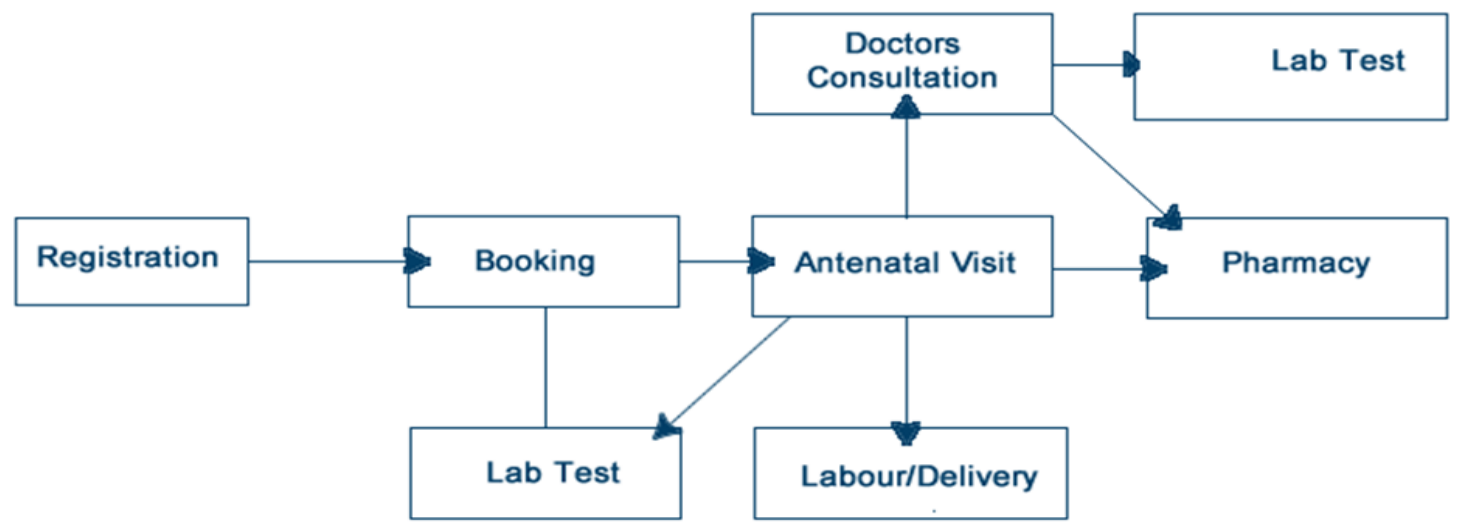

Figure 2 - Process Flow of the Application 


\section{SYSTEM INTERFACES}

The Antenatal and Postnatal system consists of different interface for different processes of antenatal and postnatal procedure of Usmanu Danfodiyo University clinic. The interfaces are
Homepages, About us, Contact us, Registration, Eresources/lecture, Antenatal booking, Antenatal service, Lab test, Vaccination, Labor/Delivery, Referral, and Reports page (Figures 3, 4).
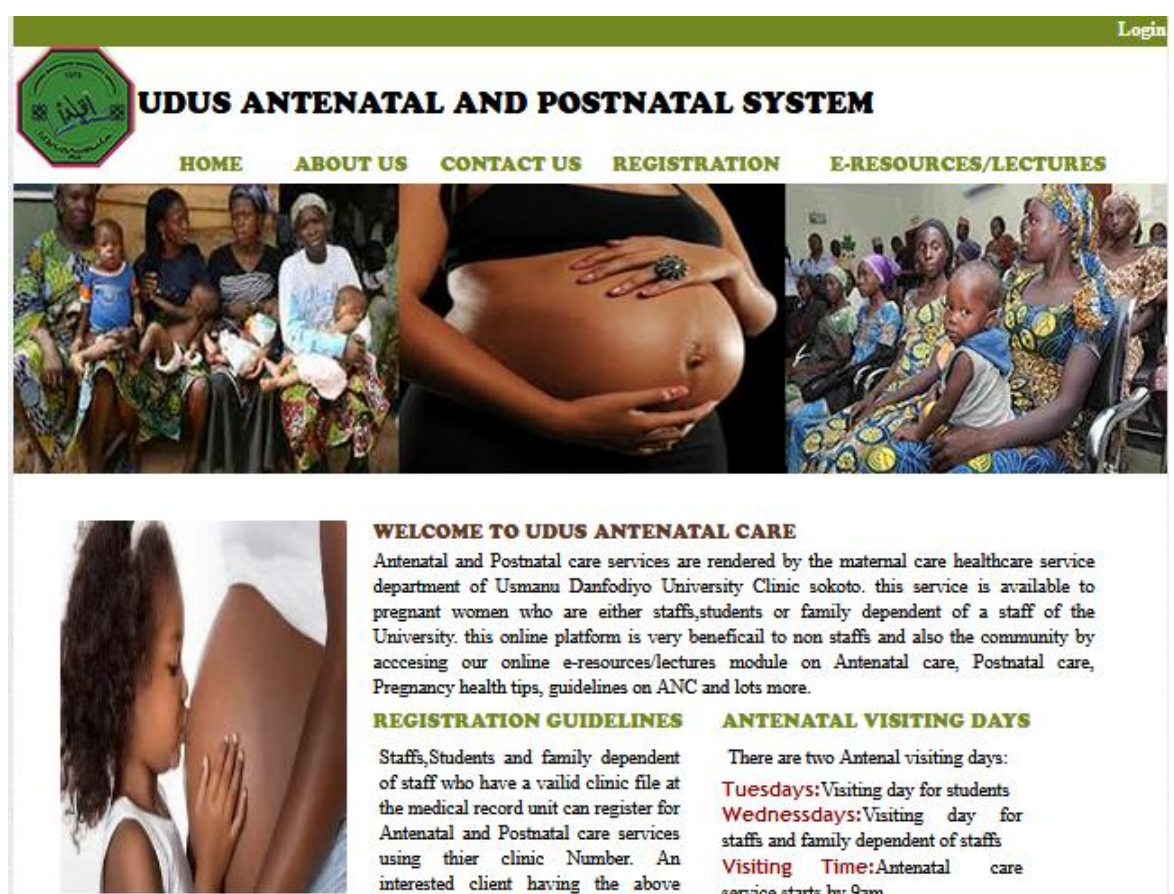

WELCOME TO UDUS ANTENATAL CARE

Antenatal and Postnatal care services are rendered by the matemal care healthcare service department of Usmanu Danfodiyo University Clinic sokoto, this service is available to department of Usmanu Danfodryo University Clinic sokoto. this service is available to University. this online platform is very beneficail to non staffs and also the community by Unrversity. this online platform is very beneficall to non stafts and also the community by
acceesing our online e-resources/lectures module on Antenatal care, Postnatal care, Pregnancy health tips, guidelines on ANC and lots more.

REGISTRATION GUIDELINES ANTENATAL VISITING DAYS

Staffs, Students and family dependent There are two Antenal visiting days: of staff who have a vailid clinic file at Tuesdays:Visiting day for students the medical record unit can register for Wednessdays:Visiting day for Antenatal and Postnatal care services staffs and family dependent of staffs using thier clinic Number. An Visiting Time:Antenatal care
interested client having the above reqirement should click on the registration link to register.

Note:A scanned passport photograph

is also required for the registration service starts by 9 am Note:Ultrasound scan for pregnant women at UDUS clinic is done on fridays by $4 \mathrm{pm}$ for pregnant women who ultrasound have been requested

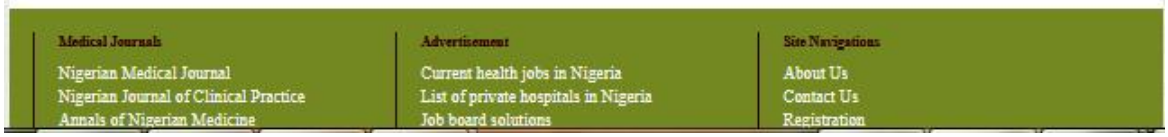

Figure 3 - Home Page

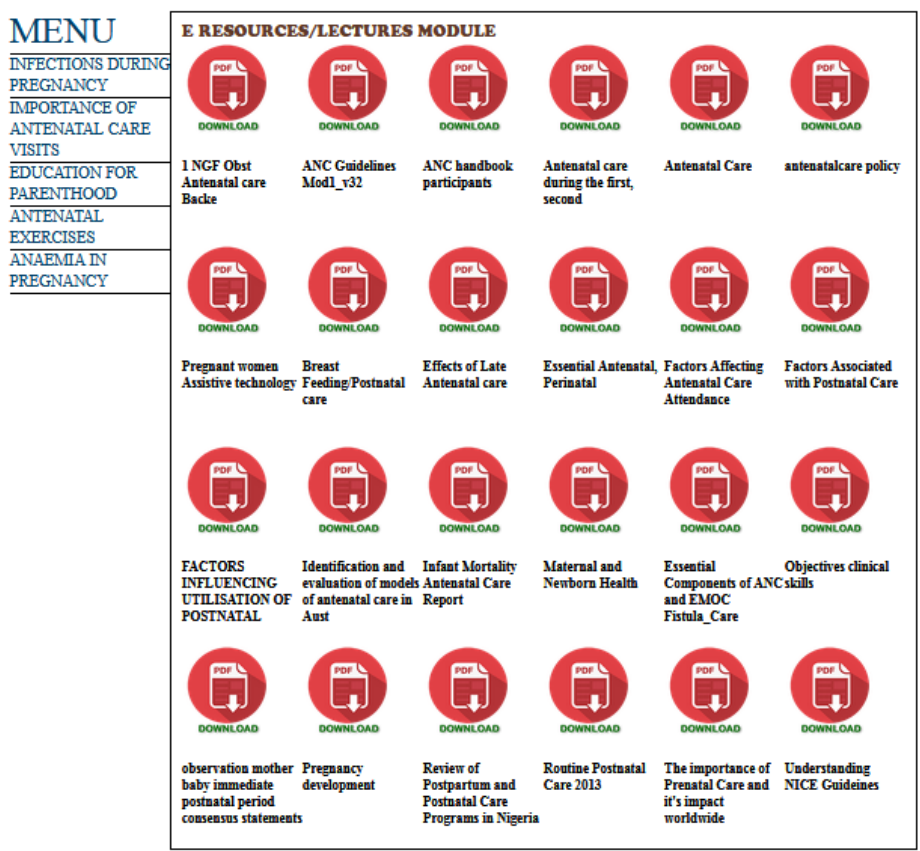

Figure 4 - E-resources 


\section{SYSTEM EVALUATION}

The integration of the antenatal and postnatal pregnancy services to the Hospital Management System is done. Then the acceptance and usefulness of the system to the target users are been evaluated based on the interaction with the system and the relevance of the services provided. This evaluation is done using some sample participants from the three selected hospitals/clinics within Sokoto metropolis.

\section{RESULTS AND DISCUSSION}

The proposed system is evaluated experimentally by collecting responses or feedback from the participants used in the study. This is achieved by analyzing the 22 SUS questionnaires filled and returned by the participants in the selected domains as the evaluation results. Eleven of the filled questionnaire was from Usmanu Danfodiyo University clinic, Ten from ANNISAH CLINIC and a consultant from specialist Hospital, Sokoto. The chart in Figure 5 shows the summary of the overall SUS assessment score given by the participants to the system developed.

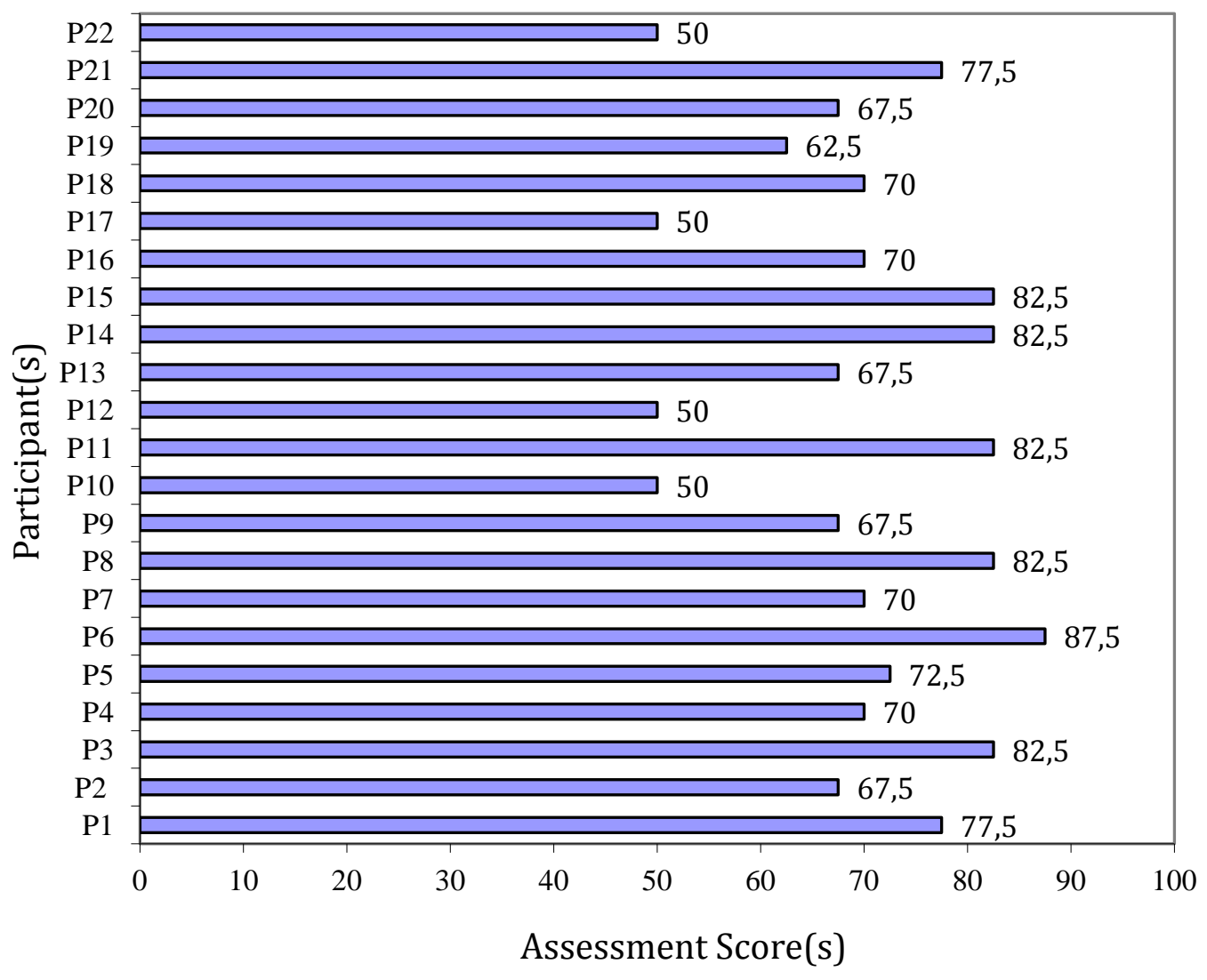

Figure 5 - Bar Chart Showing Assessment Scores of the Corresponding Participants

It is now clear from the above results obtained with SUS score an average of 70 that the target audiences are satisfied and impressed with the performance and improvement of the new system. The results show that the switch from the existing system to new system will go smoothly and the system won't be rejected by the target audience but will be embraced based on the results obtained in this study.

\section{CONCLUSION}

The antenatal and postnatal system is developed for the Usmanu Danfodiyo University Clinic. The developed system was tested and the results indicated that the new antenatal and postnatal system will be very helpful and useful to UDUS clinic and other clinics like specialist and ANNISAH. The new system can be used in any clinic or hospital to manage antenatal and postnatal care services efficiently. The system will greatly reduce data inconsistency and errors made with the 
manual system. Records in the existing system are been kept and stored in files in the existing system. In many cases, files of clients often get missing or lost which means that the client's records have been lost. The users of the system expressed their opinion and belief that if the new system is used effectively, the loss of files or records will no longer be an issue.

As a future work, the UDUS antennal system can be improved by building a central Antenatal and postnatal system for the state where several hospitals and clinics can plug-in and use instead of having an independent and decentralized implementation by individual hospitals and clinics. This will enable the government to have a general report and statistics of antenatal and postnatal services in the state. Hospitals and clinics can share data, clients records etc. The new system only stores referral records of clients. The referred hospital can't log in to the system to view referrals, take necessary actions and submit results back to the primary health clinic. With the centralized system, clinics will be able to view and act on referrals.

\section{REFERENCES}

1. Ismail, N. I., Abdullah, N. H., \& Shamsuddin, A. (2015). Adoption of Hospital Information System (HIS) in Malaysian Public Hospitals. Procedia - Social and Behavioral Sciences, 172, 336-343. doi: 10.1016/j.sbspro.2015.01.373

2. Mukherjee, C., Gupta, K., \& Nallusamy, R. (2012). A System to Provide Primary Maternity Healthcare Services in Developing Countries. 2012 Annual SRII Global Conference. doi: 10.1109/srii.2012.34

3. Niche. (2015, February). Postnatal care up to 8 weeks after birth. Retrieved from https://www.nice.org.uk/guidance/cg37

4. Onashoga, S. A., Sodiya, A. S., Omilani, T. O., \& Ajisegiri, H. O. (2011). A Mobile Phone-Based Antenatal Care Support System. 2011 21st International Conference on Systems Engineering. doi: 10.1109/icseng.2011.81

5. Parvin, S. (2015). An Effective m-Health System for Antenatal and Postnatal Care in Rural Areas of Bangladesh. IOSR Journal of Computer Engineering, 17(5), 15-19.

6. Shaikh, F. (2014). Antenatal Care. Retrieved September 1, 2018, from https://vdocuments.mx/antenatal-care-dr-fouzia-shaikh-associate-professor-lumhs.html

7. Souley, B., Usman, A., Abdulhamid, M., Zakariyau, B., \& Lookman, B. (2016). Mobile Devices Enable Antenatal Care in Remote Areas of Nigeria. British Journal of Mathematics \& Computer Science, 15(5), 1-23. doi: 10.9734/bjmcs/2016/28418 